\title{
Unravelling the Pathogenesis of Severe COVID-19 Pneumonia: Are There Possible Insights From High Altitude?
}

\author{
Gerard Thomas Flaherty ${ }^{1,2^{*}}$, Paul Hession ${ }^{3}$ \\ ${ }^{1}$ School of Medicine, National University of Ireland Galway, Galway, Ireland \\ ${ }^{2}$ School of Medicine, International Medical University, Kuala Lumpur, Malaysia \\ ${ }^{3}$ Department of Anaesthesia and Intensive Care, Cork University Hospital Group, Cork, Ireland
}

Corresponding Author: Gerard Thomas Flaherty, Professor, MD, FISTM, School of Medicine, National University of Ireland Galway, Galway, Ireland. Tel: +353-91495469, Email: gerard.flaherty@nuigalway.ie

Received March 17, 2020; Accepted April 28, 2020; Online Published April 30, 2020

Citation: Flaherty GT, Hession P. Unravelling the pathogenesis of severe COVID-19 pneumonia: are there possible insights from high altitude?. Int J Travel Med Glob Health. 2020;8(2):89-90. doi:10.34172/ijtmgh.2020.14.

\section{Dear Editor,}

The global pandemic of coronavirus disease 2019 (COVID-19) continues to exact a heavy toll on human lives, fragile healthcare systems and international travel. Following its initial rapid spread via international travel routes, ${ }^{1}$ sustained community transmission is now the major route of infection worldwide. High rates of severe pneumonia requiring prolonged ventilation, pre-symptomatic viral shedding and a disproportionate burden of mortality in older populations, are among the most challenging features of this novel coronavirus infection. A clearer profile of the clinical evolution of the disease is beginning to emerge. Gaps in our understanding of its pathogenesis compromise efforts to deliver targeted therapies to the most severely ill patients, however. The authors have, between them, managed critically ill patients with COVID-19 disease and high altitude pulmonary oedema (HAPE). Arguments have recently been advanced in the medical literature to suggest that COVID-19 lung injury may share a common pathophysiology with $\mathrm{HAPE}^{2}$ and, indeed, intriguing similarities do exist in their clinical presentation. Here we explore the points of comparison and contrast between these discrete conditions.

HAPE was regarded historically by Andean residents as the "pneumonia of the mountains". Symptoms of HAPE and COVID-19 overlap, with an initial dry cough, fever, dyspnoea and chest tightness. Also more common in males, severely affected HAPE patients are typically tachycardic, tachypnoeic and markedly hypoxaemic. Patchy nodular infiltrates on chest radiography and peripheral ground-glass opacities on CT imaging are features of both conditions, although they may also be observed in other respiratory diseases causing a non- cardiogenic pulmonary oedema. ${ }^{4}$ Acute respiratory distress syndrome (ARDS) complicates the most severe cases of COVID-19 pneumonia and is also described in severe HAPE.

The cytokine storm of the host inflammatory immune response to SARS-CoV-2 infection is central to the development of severe illness in a subset of patients, with the worst clinical outcomes reported in male patients, the elderly and in those with pre-existing illness. Anecdotally, adverse clinical outcomes are also observed more frequently in obese patients. High altitude travellers with a brisk hypoxic pulmonary vasoconstrictor response (HPVR) and risk factors for pulmonary hypertension, including obesityhypoventilation syndrome, may develop uneven pulmonary arteriolar vasoconstriction. This leads to stress failure of pulmonary capillaries in over-perfused areas of the lung, leading to a protein-rich pulmonary oedema. Evidence of alveolar epithelial inflammation manifests in both conditions. The role of defective reabsorption of sodium and water from the alveoli in HAPE led to the use of the inhaled beta-2agonist salmeterol in its prophylaxis, but to the best of our knowledge a similar approach has not been taken to date in COVID-19 patients.

Microthrombi are reported in the pulmonary vessels of patients with COVID-19, having long been associated with HAPE, although in HAPE they may be an epiphenomenon, along with the acute inflammation described later in the course of HAPE. Intriguingly, the presence of a preceding viral respiratory tract infection has been recognised in HAPE. While viral infection may lower the threshold for HAPE, it is not a sine qua non for the development of oedema. HAPE appears to be a unique example of lung injury, with

Copyright $(\odot 2020$ The Author(s). This is an open-access article distributed under the terms of the Creative Commons Attribution License (http:// creativecommons.org/licenses/by/4.0), which permits unrestricted use, distribution, and reproduction in any medium, provided the original work is properly cited. 
pulmonary arterial hypertension secondary to pulmonary vasoconstriction at its core. Whether this is also the case for COVID-19 ARDS patients appears unlikely at this point.

The individual susceptibility of patients to severe COVID-19 disease parallels a similar scenario at altitude where only certain visitors are maladapted to its hypobaric hypoxia. Unfortunately, there is no predictive test which can be applied at sea level to lowlanders embarking on a high altitude trek. Genetic variation is a plausible mechanism and, in the case of HAPE, research has focused on polymorphisms in the angiotensin converting enzyme (ACE) gene. ${ }^{5}$ Given the interaction of SARS-CoV-2 with ACE-2 receptors on respiratory epithelial cells, linkage analysis of ACE-2 gene polymorphisms may yet enhance our understanding of how genetic factors influence host sensitivity to severe COVID-19 respiratory disease.

HAPE responds well to oxygen, early descent, and portable hyperbaric chamber therapy, with the adjunctive use of nifedipine and tadalafil as pulmonary vasodilators in some cases. ${ }^{6}$ Acetazolamide attenuates the HPVR at high doses but has a dubious role in HAPE management. Inhaled nitric oxide has been used in both severe COVID-19-related ARDS and, experimentally, in HAPE patients. Prone positioning has become a mainstay in the treatment of critically ill patients with COVID-19 disease, but has not been formally evaluated in the management of HAPE. Whether therapeutic strategies in both conditions will converge remains to be seen but the weight of evidence, for now at least, argues against a robust pathophysiologic association between COVID-19 pneumonia and "pneumonia of the mountains". Further research is urgently needed to elucidate the pathogenesis of COVID-19related respiratory failure. Knowledge of how it resembles and differs from aspects of HAPE may be helpful in enhancing our understanding of how to manage the most severely affected COVID-19 patients.

\section{Authors' Contributions}

GTF conceived the idea for the manuscript. Both GTF and $\mathrm{PH}$ researched the material. GTF prepared the first draft of the manuscript which was edited for significant intellectual content by PH. Both authors approved the final version of the manuscript.

\section{Conflict of Interest Disclosures}

The authors declare that they have no conflicts of interest.

\section{Ethical Approval}

Not applicable.

\section{Funding/Support}

None.

\section{References}

1. Khedmat L. New coronavirus (2019-nCoV): an insight toward preventive actions and natural medicine. Int J Travel Med Glob Health. 2020;8(1):44-45. doi:10.34172/ijtmgh.2020.07.

2. Solaimanzadeh I. Acetazolamide, nifedipine and phosphodiesterase inhibitors: rationale for their utilization as adjunctive countermeasures in the treatment of coronavirus disease 2019 (COVID-19). Cureus. 2020;12(3):e7343. doi:10.7759/cureus.7343.

3. Hultgren $\mathrm{H}$. High altitude pulmonary edema. In: Hultgren $\mathrm{HN}$, ed. High Altitude Medicine. Stanford, California: Hultgren Publications; 1997:256-320.

4. Cao Y, Liu X, Xiong L, Cai K. Imaging and clinical features of patients with 2019 novel coronavirus SARS-CoV-2: A systematic review and meta-analysis. J Med Virol. 2020. doi:10.1002/ jmv.25822.

5. Wu AL, Xiong YS, Li ZQ, Liu YG, Quan Q, Wu LJ. Correlation between single nucleotide polymorphisms in hypoxia-related genes and susceptibility to acute high-altitude pulmonary edema. Genet Mol Res. 2015;14(3):11562-11572. doi:10.4238/2015. September.28.8.

6. Flaherty GT, Kennedy KM. Preparing patients for travel to high altitude: advice on travel health and chemoprophylaxis. $\mathrm{Br} J$ Gen Pract. 2016;66(642):e62-64. doi:10.3399/bjgp16X683377. 